\title{
ALEXANDER IDEALS OF CLASSICAL KNOTS
}

\author{
C. Kearton and S. M. J. Wilson
}

Abstract

The Alexander ideals of classical knots are characterised, a result which extends to certain higher dimensional knots.

\section{Introduction}

Let $K$ be the closed complement of a tubular neighbourhood of a classical knot $k=\left(S^{3}, S^{1}\right)$. By theorems of Hurewicz and Alexander, $\pi_{1}(K, *)$ abelianises to $H_{1}(K)$ which is isomorphic to the infinite cyclic group $(t:)$, written multiplicatively. This yields the infinite cyclic cover $\tilde{K} \rightarrow K$, and hence we obtain $H_{1}(\tilde{K})$ as a finitely generated module over the ring $\Lambda=Z\left[t, t^{-1}\right]$. This module has associated with it a sequence of ideals of $\Lambda$,

$$
E_{1} \subseteq E_{2} \subseteq E_{3} \subseteq \cdots \subseteq E_{n}=E_{n+1}=\cdots=\Lambda .
$$

The highest common factor of $E_{i}$ is a Laurent polynomial $\Delta_{i}(t)$, defined up to multiplication by $\pm t^{r}$. These are known as the $i$ th Alexander ideal and $i$ th Alexander polynomial of the knot.

Definition 1.1. Let $A_{i}$ be the set of ideals of $\Lambda$ which arise as the $i$ th Alexander ideal of some knot, and let $A=\bigcup_{i=1}^{\infty} A_{i}$.

It is shown in Corollary 2.3 below that

$$
A_{1} \subset A_{2} \subseteq A_{3} \subseteq \cdots \subseteq A .
$$

The first author wishes to thank the Government of Catalunya and the Centre de Recerca Matemàtica of the Institut d'Estudis Catalans at the Universitat Autònoma de Barcelona for their support and hospitality.

Keywords. Alexander ideal, knot.

1991 Mathematics subject classifications: 57M25, 57Q45. 
The first inclusion is strict, since all the ideals in $A_{1}$ are principal whereas those in $A_{2}$ are not necessarily so (see [1] for examples).

A polynomial $f(t) \in \Lambda$ arises as $\Delta_{i}(t)$ for some $i$ and some knot $k$ if and only if $f(1)= \pm 1$ and $f\left(t^{-1}\right)= \pm t^{r} f(t)$ for some $r$ (see [3] and [5]). The main result of this paper, Theorem 2.5, gives a similar characterisation of the ideals belonging to $A$.

\section{Results}

For us an Alexander matrix $U$ of a knot will be one which presents $H_{1}(\tilde{K})$ as a module over $\Lambda$. We shall assume that it is square; indeed, we could assume that $U=t V-V^{\prime}$ where $V$ is a Seifert matrix of the knot.

Definition 2.1. Let $U$ be an $m \times n$ matrix with entries in $\Lambda$. For each integer $k \geq 1$, the $k$ th elementary ideal $E_{k}(U)$ is defined as follows.

(1) If $0 \leq n-k<m$, then $E_{k}(U)$ is the ideal generated by the $(n-k+1) \times(n-k+1)$ minors of $U$.

(2) If $n-k \geq m$, then $E_{k}(U)=(0)$.

(3) If $n-k<0$, then $E_{k}(U)=\Lambda$.

Note that this differs slightly from the definition of elementary ideal given in [1] , because our Alexander matrices are square. As shown in [1], the elementary ideals of $U$ form an ascending chain

$$
E_{1}(U) \subseteq E_{2}(U) \subseteq \cdots \subseteq E_{n}(U)=E_{n+1}(U)=\cdots=\Lambda .
$$

If $U$ is an Alexander matrix of a knot then, of course, the sequence of elementary ideals of $U$ depends only on the knot, and is the sequence of Alexander ideals.

Lemma 2.2. Let $U$ be an $n \times n$ matrix with entries in $\Lambda$. Then for every integer $r>0$ there is an $(n+r) \times(n+r)$ matrix $V$ such that the sequence

$$
E_{1+r}(V) \subseteq E_{2+r}(V) \subseteq \cdots \subseteq E_{n+r}(V)
$$

is the same as

$$
E_{1}(U) \subseteq E_{2}(U) \subseteq \cdots \subseteq E_{n}(U) .
$$

Proof: Let $\Delta=\operatorname{det} U$, and first consider the case $r=1$. Let

$$
V=\left(\begin{array}{cc}
U & 0 \\
0 & \Delta
\end{array}\right)
$$


so that $V$ is an $(n+1) \times(n+1)$ matrix. For $1 \leq s \leq n, E_{s+1}(V)$ is generated by the $(n-s+1) \times(n-s+1)$ minors of $V$, and the only possible non-zero ones are the $(n-s+1) \times(n-s+1)$ minors of $U$ and ones of the form $\Delta$ times an $(n-s) \times(n-s)$ minor of $U$. Since $\Delta \in(\Delta)=E_{1}(U) \subseteq E_{s}(U)$, these generate $E_{s}(U)$. This establishes the result for $r=1$; repeated application of the argument gives the general case.

\section{Corollary 2.3.}

$$
A_{1} \subset A_{2} \subseteq A_{3} \subseteq \cdots \subseteq A
$$

Proof: If $U$ is an Alexander matrix, then $(\operatorname{det} U)$ is also an Alexander matrix, hence so is $V$ in the proof above.

Define the map $\varepsilon: \Lambda \rightarrow Z$ to be the ring homomorphism sending $t \mapsto 1$, and define conjugation in $\Lambda$ to be the ring homomorphism sending $t \mapsto t^{-1}$, denoted $\bar{t}$. If $U$ is the Alexander matrix of a classical knot, then the following properties of $E_{i}(U)$ are well known (see [1]).

$$
\begin{aligned}
\overline{E_{i}(U)} & =E_{i}(U), \\
\varepsilon\left(E_{i}(U)\right) & =Z
\end{aligned}
$$

for all $i \geq 1$.

Lemma 2.4. Let $g \in \Lambda$ satisfy $g(1)=1$. Then there is a classical knot $k$ which has

$$
H_{1}(\tilde{K}) \cong \frac{\Lambda}{(g)} \oplus \frac{\Lambda}{(\bar{g})}
$$

Proof: Let $M$ be the module above, generated by $u, v$ such that $g u=$ $0=\bar{g} v$. Define an hermitian pairing

$$
(,): M \times M \rightarrow \Lambda_{o} / \Lambda
$$

where $\Lambda_{o}$ is the field of fractions of $\Lambda$, by

$$
\begin{aligned}
& (u, v)=\frac{1}{g}=\overline{(v, u)}, \\
& (u, u)=0=(v, v) .
\end{aligned}
$$

The map $(1-t): M \rightarrow M$ is an isomorphism. In fact,

$$
(g(1)-g(t)) u=g(1) u-g(t) u=u
$$


and

$$
g(1)-g(t)=(1-t) a(t)
$$

for some $a(t) \in \Lambda$. A similar argument holds for $v, \bar{g}$; hence the map is onto. Suppose that $(1-t) w=0$. Then $t w=w$, and so

$$
\begin{aligned}
& g(t) w=g(1) w=w \\
& \bar{g}(t) w=\bar{g}(1) w=w .
\end{aligned}
$$

But $\bar{g}(t) g(t)$ annihilates $M$, so $0=\bar{g}(t) g(t) w=\bar{g}(t) w=w$, and hence the map is one-one.

The pairing defined above is nonsingular, in the sense that the adjoint map

$$
\theta: M \rightarrow \overline{\operatorname{Hom}}\left(M, \Lambda_{o} / \Lambda\right)
$$

is an isomorphism. To see this, suppose that $\theta(w)=0$, where $w=$ $a u+b v$. Then

$$
0=(a u+b v, u)=\frac{b}{\bar{g}} \in \Lambda_{o} / \Lambda
$$

and so $b=\beta \bar{g}$ for some $\beta \in \Lambda$. Thus $w=a u+b v=a u+\beta \bar{g} v=a u$. Now

$$
0=(a u, v)=\frac{a}{g} \in \Lambda_{o} / \Lambda
$$

and so $a=\alpha g$ for some $\alpha \in \Lambda$. Thus $w=a u=\alpha g u=0$. Hence $\theta$ is a monomorphism.

Let $f \in \overline{\operatorname{Hom}}\left(M, \Lambda_{o} / \Lambda\right)$, and let $a, b \in \Lambda$ satisfy

$$
f(u)=\frac{a}{\bar{g}}, \quad f(v)=\frac{b}{g} .
$$

Then

$$
\begin{aligned}
& (b u+a v, u)=a(v, u)=\frac{a}{\bar{g}}=f(u) \\
& (b u+a v, v)=b(u, v)=\frac{b}{g}=f(v)
\end{aligned}
$$

and $\operatorname{so} \theta(b u+a v)=f$.

It is shown in [2] (or [4], or [6]) that any finitely generated $\Lambda$-torsionmodule $M$ for which multiplication by $1-t$ is an isomorphism and which supports a nonsingular hermitian pairing arises as $H_{1}(\tilde{K})$ for some classical knot. 
Theorem 2.5. Let $E$ be an ideal of $\Lambda$. Then $E \in A$ if and only if $E$ satisfies $\bar{E}=E$ and $\varepsilon(E)=Z$.

Proof: As remarked above, the necessity is well known. So suppose that $E$ satisfies the two conditions. By the Hilbert Basis Theorem, the ideal $E \cap Z[t]$ of $Z[t]$ is finitely generated over $Z[t]$. A set of generators of $E \cap Z[t]$ over $Z[t]$ is clearly a set of generators of $E$ over $Z\left[t, t^{-1}\right]$, so $E$ is finitely generated, say $E=\left(f_{1}(t), \ldots, f_{n}(t)\right)$.

We claim that $E$ has a set of generators $g_{1}(t), \ldots, g_{n}(t)$ with $g_{1}(1)=\cdots=g_{n}(1)=1$. For not all of the $f_{i}(1)$ can be zero, since $\varepsilon(E)=Z$. Multiplying by -1 if necessary, we can renumber so that

$$
f_{1}(1) \geq f_{2}(1) \geq f_{3}(1) \geq \cdots \geq f_{n}(1) \geq 0 .
$$

If $f_{n}(1)=0$, then replace this set of generators by $f_{1}(t), \ldots, f_{n-1}(t)$, $f_{1}(t)+f_{n}(t)$. Repeat this process of replacement and renumbering until we have a set of generators $f_{1}(t), \ldots, f_{n}(t)$ with

$$
f_{1}(1) \geq f_{2}(1) \geq f_{3}(1) \geq \cdots \geq f_{n}(1)>0 .
$$

If they all evaluate to 1 , well and good. If not, we must have $f_{1}(1)>$ $f_{n}(1)$, since $\varepsilon(E)=Z$. Replace $f_{1}(t)$ by $f_{1}(t)-f_{n}(t)$. If the new set of generators is denoted by $f_{1}^{\prime}(t), \ldots, f_{n}^{\prime}(t)$, then $f_{i}^{\prime}(1)>0$ for all $i$ and

$$
n \leq \sum_{i=1}^{n} f_{i}^{\prime}(1)<\sum_{i=1}^{n} f_{i}(1) \text {. }
$$

Continuing in this way we arrive at the desired set of generators $g_{1}(t), \ldots, g_{n}(t)$.

For each $g_{i}$, let $k_{i}$ be the knot whose existence is guaranteed by Lemma 2.4 , and let $k=k_{1}+\cdots+k_{n}$. Then $H_{1}(\tilde{K})$ is the orthogonal direct sum of the $H_{1}\left(\tilde{K}_{i}\right)$, and has a diagonal Alexander matrix $U$ with entries $g_{1}, \bar{g}_{1}, \ldots, g_{n}, \bar{g}_{n}$ on the diagonal. Clearly $E_{2 n}(U)=E$.

Remark. Using the results of [2] (or [4], or [6]), we see that the set of Alexander ideals is the same for each set of simple $(4 q+1)$-knots, $q=0,1,2, \ldots$, since the set of modules and pairings which arise is the same in each dimension. This extends easily to the simple $(4 q-1)$ knots, $q=1,2, \ldots$, by defining a skew-hermitian pairing instead of an hermitian pairing in the proof of Lemma 2.4, and noting that the form of the pairing ensures that the signature of the associated quadratic form is zero (this is needed for the case $q=1$, where the signature must be a multiple of 16 ). 


\title{
3. Questions
}

As mentioned in the Introduction, it is known that $A_{1} \neq A_{2}$. Is it true that $A_{n} \neq A_{n+1}$ for all $n$ ? For finitely many $n$ ? Only for $n=1$ ? Can one characterise $A_{n}$ ? And what of the sequence of ideals? Can any sequence be realised, subject to each $E_{i}$ satisfying the two conditions known to be necessary? The corresponding question for Alexander polynomials has been answered in the affirmative by Levine in $[\mathbf{3}]$.

\section{References}

1. R. H. Crowell and R. H. Fox, "Introduction to Knot Theory," Springer-Verlag, New York, 1963.

2. C. Kearton, Classification of simple knots by Blanchfield duality, Bull. Amer. Math. Soc. 79 (1973), 952-955.

3. J. Levine, A characterization of knot polynomials, Topology 4 (1965), 135-141.

4. J. Levine, Knot modules, Trans. Amer. Math. Soc. 229 (1977), $1-50$.

5. H. SeIfert, Über das Geschlect von Knoten, Math. Ann. 110 (1934), 571-592.

6. H. F. Trotter, Knot modules and Seifert matrices, in "Knot Theory, Proceedings, Plans-sur-Bex 1977," Lecture Notes in Math. 685, Springer-Verlag, New York, 1978, pp. 291-299.

\author{
Department of Mathematics \\ Durham University \\ South Road \\ Durham, DH1 3LE \\ ENGLAND \\ e-mail: Cherry.Kearton@durham.ac.uk \\ e-mail: S.M.J.Wilson@durham.ac.uk
}

Primera versió rebuda el 6 de Maig de 1996 , darrera versió rebuda el 3 de Setembre de 1996 